MATHEMATICS OF COMPUTATION

Volume 67, Number 224, October 1998, Pages 1451-1471

S $0025-5718(98) 01014-\mathrm{X}$

\title{
A COMPARISON OF REGULARIZATIONS FOR AN ILL-POSED PROBLEM
}

\author{
KAREN A. AMES, GORDON W. CLARK, JAMES F. EPPERSON, \\ AND SETH F. OPPENHEIMER
}

\begin{abstract}
We consider numerical methods for a "quasi-boundary value" regularization of the backward parabolic problem given by

$$
\left\{\begin{array}{l}
u_{t}+A u=0, \quad 0<t<T \\
u(T)=f
\end{array}\right.
$$

where $A$ is positive self-adjoint and unbounded. The regularization, due to Clark and Oppenheimer, perturbs the final value $u(T)$ by adding $\alpha u(0)$, where $\alpha$ is a small parameter. We show how this leads very naturally to a reformulation of the problem as a second-kind Fredholm integral equation, which can be very easily approximated using methods previously developed by Ames and Epperson. Error estimates and examples are provided. We also compare the regularization used here with that from Ames and Epperson.
\end{abstract}

\section{INTRODUCTION}

Let $A$ be a self-adjoint operator on a Hilbert space $H$ such that $-A$ generates a compact contraction semi-group on $H$, and 0 is in the resolvent set of $-A$; perhaps the canonical example is $A=-\Delta$, the Laplace operator. We consider numerical solutions to the problem: find $u:[0, T] \longrightarrow H$ such that

$$
\left\{\begin{array}{l}
u^{\prime}(t)+A u(t)=0, \quad 0<t<T \\
u(T)=f
\end{array}\right.
$$

for some prescribed final value $f$ in $H$. Such problems are not well posed; that is, even if a unique solution exists on $[0, T]$, it need not depend continuously on the final value $f$.

In the context of analytic approximation for this problem, many approaches have been tried. Such authors as Lattes and Lions [8], Miller [10], and Showalter [13] have approximated (FVP) by perturbing the operator $A$. In their work, Clark and Oppenheimer [4] followed what Showalter [14] did in a more general context, and approximated (FVP) by perturbing the final value condition. This yields the quasi-boundary value problem

(QBVP)

$$
\left\{\begin{aligned}
u_{\alpha}^{\prime}(t)+A u_{\alpha}(t) & =0, \quad 0<t<T \\
\alpha u_{\alpha}(0)+u_{\alpha}(T) & =f,
\end{aligned}\right.
$$

Received by the editor April, 17, 1996.

1991 Mathematics Subject Classification. Primary 35A35, 35R25, 65M30, 65M15.

Key words and phrases. Quasi-reversibility, final value problems, ill-posed problems, Freholm equations, numerical methods.

Partially supported by Army contract DACA 39-94-K-0018 (S.F.O.) and by NSF contract DMS-9308121 (K.A.A.).

(C)1998 American Mathematical Society 
where $\alpha$ is a small positive number.

As is shown in [4], this approximation has many nice properties, some of which we will need here. The operator $A$ has an orthonormal set of eigenfunctions $q_{i}$, with eigenvalues $\lambda_{i}>0$, such that the set $\left\{q_{i}\right\}$ forms an eigenbasis for $H$. We will always take the $f$ appearing in (FVP) to have an expansion in this eigenbasis given by $f=\sum_{i=1}^{\infty} b_{i} q_{i}$. It is the use of this eigenstructure that gives the precise estimates in [4] and distinguishes the approach there from that found in [14].

Our goal in this paper is to implement the abstract approximation scheme of Clark and Oppenheimer numerically. Of course, before we can ask questions about the implementation and accuracy of an approximation, we must first answer the question: Is there anything to approximate? The answer is yes if and only if $\sum_{i=1}^{\infty} b_{i}^{2} e^{2 T \lambda_{i}}$ converges. The two theorems from [4] which are most important in the context of this paper bear directly on this question and are given below.

Theorem 1. For all $f$ in H, (FVP) has a solution $u$ if and only if the sequence $u_{\alpha}(0)$ converges in $H$. Furthermore, we then have that $u_{\alpha}(t)$ converges to $u(t)$ as $\alpha$ tends to zero, uniformly in $t$.

Theorem 2. If $f=\sum_{i=0}^{\infty} b_{i} q_{i}$ is in $H$ and there exists a positive $\epsilon$ so that

$$
s_{\epsilon, T}=\sum_{i=0}^{\infty} b_{i}^{2} e^{(2+\epsilon) \lambda_{i} T}
$$

converges, then $\left\|u_{\alpha}(t)-u(t)\right\|$ converges to zero with order $\alpha^{\epsilon} \epsilon^{-2}$, uniformly in $t$.

Theorem 1 guarantees us that it is worth while to look for a numerical implementation, and Theorem 2 gives us an upper bound on the convergence rate we can expect. Theorem 2 will be re-stated more explicitly and slightly improved in the next section.

It is interesting to note that even when (FVP) fails to have a solution, the regularized solution at $t=T$ still converges to $f$. Formally, we have:

Theorem 3. For all $f \in H,\left\|u_{\alpha}(T)-f\right\|$ converges to zero as $\alpha \rightarrow 0$.

This is Theorem 3 of [4]. Note that there is no convergence rate.

We close this section with some notation and assumptions that will apply for the rest of the paper.

We assume that $-A$ is a uniformly elliptic differential operator on a bounded, open domain $\Omega$, with homogeneous Dirichlet data imposed on $\partial \Omega$, and we take our Hilbert space $H$ to be $L^{2}(\Omega)$. Associated with the operator $A$ is the bilinear form $a: H_{0}^{1}(\Omega) \times H_{0}^{1}(\Omega) \rightarrow \mathbf{R}$ such that

$$
-A v=g
$$

if and only

$$
a(v, w)=(g, w) \quad \forall w \in H_{0}^{1}(\Omega) .
$$

We assume that a solution to (FVP) actually exists and satisfies the estimate

$$
\|u(t)\| \leq m
$$

for all $t \in[0, T]$. Here, and in what follows, $\|\cdot\|$ will denote the $L^{2}(\Omega)$ norm, and $\|\cdot\|_{r}$ the $H^{r}(\Omega)$ norm. 


\section{REFORMULATION OF THE PROBLEM AND REGULARIZATION ESTIMATES}

In this section we will show that (QBVP) can be written as a Fredholm integral equation of the second kind. This will permit the application of approximation schemes found in [1].

Let $S(t)$ be the compact contraction semi-group generated by $-A$. Then we have that

$$
u_{\alpha}(t)=S(t) u_{\alpha}(0)
$$

or, solving for $u_{\alpha}(0)$ in terms of $f$,

$$
u_{\alpha}(t)=S(t)(\alpha I+S(T))^{-1} f .
$$

Hence,

$$
\alpha u_{\alpha}(t)+S(T) u_{\alpha}(t)=S(t) f,
$$

which, for fixed $t$, is a Fredholm integral equation of the second kind. To see this more clearly, let $K$ be the kernel of the operator $\partial_{t}+A$ so that we have

$$
v(t)=S(t) v(0)
$$

if and only if

$$
v(x, t)=\int_{\Omega} K(x, t, \xi) v(\xi, 0) d \xi
$$

Therefore, we have

$$
\alpha u_{\alpha}(x, t)+\int_{\Omega} K(x, T, \xi) u_{\alpha}(\xi, t) d \xi=F(x, t),
$$

where

$$
F(x, t)=\int_{\Omega} K(x, t, \xi) f(\xi) d \xi .
$$

We adopt the point of view that we are interested in approximating $u$ only at the specific time $t=t_{*}$, i.e., we are only interested in computing $u_{\alpha}\left(t_{*}\right)$. For notational simplicity denote $\phi=u\left(t_{*}\right)$ and $\phi_{\alpha}=u_{\alpha}\left(t_{*}\right)$. We therefore have that $\phi_{\alpha}$ satisfies the second kind Fredholm equation

$$
\alpha \phi_{\alpha}(x)+\int_{\Omega} K(x, T, \xi) \phi_{\alpha}(\xi) d \xi=F\left(x, t_{*}\right) .
$$

For notational simplicity we write this in operator form as

$$
\alpha \phi_{\alpha}+\mathcal{K}_{1} \phi_{\alpha}=\mathcal{K}_{2} f
$$

where

$$
\mathcal{K}_{1} v=\int_{\Omega} K(\cdot, T, \xi) v(\xi) d \xi
$$

and

$$
\mathcal{K}_{2} v=\int_{\Omega} K\left(\cdot, t_{*}, \xi\right) v(\xi) d \xi
$$

Since $\phi=u\left(t_{*}\right)$ the regularization estimate from Theorem 2 becomes

$$
\left\|\phi-\phi_{\alpha}\right\| \leq C \alpha^{\epsilon} \epsilon^{-2}
$$

where $\|\cdot\|$ is the $L^{2}(\Omega)$ norm and we assume that $\sum_{i=0}^{\infty} b_{i}^{2} e^{(2+\epsilon) \lambda_{i} T}$ converges. We can improve this, slightly, as follows: 
Theorem 4. If $f=\sum_{i=0}^{\infty} b_{i} q_{i}$ is in $H=L^{2}(\Omega)$ and there exists $\epsilon \in(0,1)$ so that $\sum_{i=0}^{\infty} b_{i}^{2} e^{2 \lambda_{i}(1+\epsilon) T}$ converges, then, for $t \geq 0$,

$$
\left\|u_{\alpha}(t)-u(t)\right\| \leq \alpha^{\epsilon} M(\epsilon) .
$$

Here

$$
M(\epsilon)=\left(\sum_{i=0}^{\infty} b_{i}^{2} e^{2(1+\epsilon) \lambda_{i} T}\right)^{\frac{1}{2}} .
$$

Moreover, if $\epsilon \leq t / T$, then

$$
\left\|u_{\alpha}(t)-u(t)\right\| \leq \alpha^{\epsilon}\|u(0)\| .
$$

Proof. Since $\sum_{i=1}^{\infty} b_{i}^{2} e^{2 T \lambda_{i}}$ converges, we can write

$$
u(t)=S(t) S(T)^{-1} f
$$

and

$$
u_{\alpha}(t)=S(t)(\alpha I+S(T))^{-1} f
$$

so that

$$
\begin{aligned}
u(t)-u_{\alpha}(t) & =S(t)\left(S(T)^{-1}-(\alpha I+S(T))^{-1}\right) f \\
& =\alpha \sum_{i=1}^{\infty} e^{\lambda_{i}(T-t)}\left(\alpha+e^{-\lambda_{i} T}\right)^{-1} b_{i} q_{i} .
\end{aligned}
$$

Therefore, for any $\epsilon \in(0,1)$

$$
\begin{aligned}
\left\|u(t)-u_{\alpha}(t)\right\|^{2} & =\alpha^{2} \sum_{i=1}^{\infty} e^{2 \lambda_{i}(T-t)} b_{i}^{2}\left(\alpha+e^{-\lambda_{i} T}\right)^{-2} \\
& =\alpha^{2} \sum_{i=1}^{\infty} e^{2 \lambda_{i}(T-t)} b_{i}^{2}\left[\left(\alpha+e^{-\lambda_{i} T}\right)^{\epsilon}\left(\alpha+e^{-\lambda_{i} T}\right)^{1-\epsilon}\right]^{-2} \\
& \leq \alpha^{2} \sum_{i=1}^{\infty} e^{2 \lambda_{i}(T-t)} b_{i}^{2}\left(e^{2 \lambda_{i} T}\right)^{\epsilon}\left(\alpha^{1-\epsilon}\right)^{-2} \\
& \leq \alpha^{2 \epsilon} \sum_{i=1}^{\infty} b_{i}^{2} e^{2 \lambda_{i}((1+\epsilon) T-t)}
\end{aligned}
$$

from which both conclusions follow immediately.

Remark. If $\epsilon>1$, then, since $\lambda_{1} \leq \lambda_{i}$,

$$
\begin{aligned}
{\left[\left(\alpha+e^{-\lambda_{i} T}\right)^{\epsilon}\left(\alpha+e^{-\lambda_{i} T}\right)^{1-\epsilon}\right]^{-2} } & =\left(\frac{1}{\alpha+e^{-\lambda_{i} T}}\right)^{2 \epsilon}\left(\alpha+e^{-\lambda_{i} T}\right)^{2 \epsilon-2} \\
& \leq e^{2 \epsilon \lambda_{i} T}\left(\alpha+e^{-\lambda_{1} T}\right)^{2 \epsilon-2}
\end{aligned}
$$

from which we still get an error bound of the form

$$
\left\|u_{\alpha}(t)-u(t)\right\| \leq c(\alpha) M(\epsilon)
$$

where $c(\alpha)=\alpha\left(\alpha+e^{-\lambda_{1} T}\right)^{\epsilon-1}$, which goes to 0 quite rapidly as $\alpha$ does. 
In particular, taking $t=t_{*}$ (and assuming for simplicity that $\epsilon \in(0,1)$ ) we have

$$
\left\|\phi-\phi_{\alpha}\right\| \leq \alpha^{\epsilon}\|u(0)\|
$$

so long as $\epsilon \leq t_{*} / T$. Alternatively, if the initial data $f$ is sufficiently smooth, in the sense that $M(1)=\left(\sum_{i=0}^{\infty} b_{i}^{2} e^{4 \lambda_{i} T}\right)^{1 / 2}$ converges, then

$$
\left\|\phi-\phi_{\alpha}\right\| \leq \alpha M(1) \text {. }
$$

Finally, we note that taking $t=0$ and $\epsilon \rightarrow 0$ yields

$$
\left\|u(0)-u_{\alpha}(0)\right\|^{2} \leq \sum_{i=1}^{\infty} b_{i}^{2} e^{2 \lambda_{i} T}=\|u(0)\|^{2} .
$$

Theorem 4 is analogous to Theorem 2.6 of [1], which was derived using the classic log-convexity argument associated with ill-posed problems. It says, roughly, that to the extent that the solution can be continued further backwards in time, then the regularization will be accurate. Similar results are in Groetsch [6]. In fact, we can modify the kind of argument used in [1] to get analogous results for our problem here.

Theorem 5. If $f \in L^{2}(\Omega)$ and $\|u(0)\| \leq m$, then, for any $t \in(0, T)$

$$
\left\|u_{\alpha}(t)-u(t)\right\| \leq m \alpha^{t / T}
$$

Proof. We use the classic log-convexity argument as given in [11]. If we subtract (FVP) and (QBVP) we get that the error $e(t)=u(t)-u_{\alpha}(t)$ satisfies

$$
\begin{aligned}
e^{\prime}(t)+A e(t) & =0, \\
e(T) & =\alpha u_{\alpha}(0) .
\end{aligned}
$$

Now define the functional

$$
g(t)=\int_{\Omega} e^{2}(t) d x
$$

for which we quickly get

$$
g^{\prime}(t)=2 a(e, e)
$$

(here $a(\cdot, \cdot)$ is the bilinear form associated with $-A$ ) and

$$
g^{\prime \prime}(t)=4 \int_{\Omega} e_{t}^{2} d x=4 \int_{\Omega}(A e)^{2} d x
$$

Thus, Schwarz's inequality implies that

$$
g g^{\prime \prime}-\left(g^{\prime}\right)^{2} \geq 0
$$

so that $g(t)$ is log-convex, from which the inequality

$$
g(t) \leq[g(0)]^{1-t / T}[g(T)]^{t / T}
$$

follows. But $g(T)=\alpha^{2}\left\|u_{\alpha}(0)\right\|^{2}$ and $g(t)=\left\|u(t)-u_{\alpha}(t)\right\|^{2}$ so we have

$$
\left\|u(t)-u_{\alpha}(t)\right\| \leq\left\|u(0)-u_{\alpha}(0)\right\|^{1-t / T}\left(\alpha\left\|u_{\alpha}(0)\right\|\right)^{t / T}
$$

or

$$
\left\|u(t)-u_{\alpha}(t)\right\| \leq \alpha^{t / T}\left\|u_{\alpha}(0)\right\|^{t / T}\left\|u(0)-u_{\alpha}(0)\right\|^{1-t / T} .
$$

But a direct calculation shows that

$$
\left\|u_{\alpha}(t)\right\| \leq\|u(t)\|
$$


uniformly in $t$, which, together with (3), implies

$$
\left\|u(t)-u_{\alpha}(t)\right\| \leq \alpha^{t / T}\|u(0)\|,
$$

and we are done.

If we take $t=t_{*}$ and define $\theta=t_{*} / T$, then Theorem 5 implies that

$$
\left\|\phi-\phi_{\alpha}\right\| \leq m \alpha^{\theta} \text {. }
$$

If our data is perturbed by some noise term, then the regularization error can be severely affected. Assume that the data is given by $f_{\delta} \approx f$, with $\left\|f-f_{\delta}\right\| \leq \delta$ assumed. Let $\phi_{\alpha}^{\delta}$ be the regularized solution corresponding to data $f_{\delta}$. Then we have that the difference $w=\phi_{\alpha}-\phi_{\alpha}^{\delta}$ satisfies

$$
\alpha w+\mathcal{K}_{1} w=\mathcal{K}_{2}\left(f-f_{\delta}\right) .
$$

Using the eigenexpansion we can explicitly solve this, getting

$$
\phi_{\alpha}-\phi_{\alpha}^{\delta}=\sum_{i=1}^{\infty} \frac{e^{-\lambda_{i} t_{*}}}{\alpha+e^{-\lambda_{i} T}}\left(f-f_{\delta}, q_{i}\right) q_{i}
$$

Therefore,

$$
\left\|\phi_{\alpha}-\phi_{\alpha}^{\delta}\right\| \leq \delta \max _{\xi \in[0,1]}\left|\frac{\xi^{\theta}}{\alpha+\xi}\right|
$$

where, as before, $\theta=t_{*} / T \leq 1$. Simple calculus then implies that

$$
\left|\frac{\xi^{\theta}}{\alpha+\xi}\right| \leq \alpha^{-(1-\theta)}
$$

from which we get

$$
\left\|\phi_{\alpha}-\phi_{\alpha}^{\delta}\right\| \leq \delta \alpha^{-(1-\theta)}
$$

which should be contrasted with the corresponding result in Ames and Epperson [1]:

$$
\left\|\phi_{\alpha}-\phi_{\alpha}^{\delta}\right\| \leq \frac{1}{2} \delta \alpha^{-1 / 2}
$$

This implies that the method studied here should be less sensitive to noise in the data for $\theta>\frac{1}{2}$, i.e., for "short-term" problems.

Our task now is to construct numerical methods to approximate $\phi_{\alpha}$. Before embarking on that effort, however, we digress slightly to make a comparison between the regularization used here and that used in [1].

\section{Comparison with the Regularization of [1]}

It is natural for us to compare the regularization discussed here with that used by Ames and Epperson in [1]. Since they worked with the initial-value problem for the backward form of the operator, the transformation $t \leftarrow T-t$ needs to be performed before a fair comparison can be made. Once that is done we have the following.

The regularization used here can be written in terms of the eigenfunction basis as

$$
\phi_{\alpha}=\sum_{i=1}^{\infty}\left(\frac{e^{-\lambda_{i} t_{*}}}{\alpha+e^{-\lambda_{i} T}}\right)\left(f, q_{i}\right) q_{i}
$$


and is accurate in the sense that (Theorem 5)

$$
\left\|\phi-\phi_{\alpha}\right\| \leq m \alpha^{\theta}
$$

for $\theta=t_{*} / T$.

The regularization used in [1] can be written as

$$
\phi_{\alpha}=\sum_{i=1}^{\infty}\left(\frac{e^{-\lambda_{i}\left(T-t_{*}\right)}}{\alpha+e^{-2 \lambda_{i}\left(T-t_{*}\right)}}\right)\left(f, q_{i}\right) q_{i}
$$

and is accurate in the sense that

$$
\left\|\phi-\phi_{\alpha}\right\| \leq C \alpha^{\tau}
$$

where here we have $\tau=t_{*} /\left(2 T-t_{*}\right)=\theta /(2-\theta)$.

Note that some manipulation with the expansion coefficients in (9) lets us write it as

$$
\phi_{\alpha}=\sum_{i=1}^{\infty}\left(\frac{e^{-\lambda_{i} t_{*}}}{\alpha e^{\lambda_{i}\left(T-2 t_{*}\right)}+e^{-\lambda_{i} T}}\right)\left(f, q_{i}\right) q_{i} .
$$

In other words, both methods can be considered as coming from the family of regularizations defined by

$$
\phi_{\alpha}=\sum_{i=1}^{\infty}\left(\frac{e^{-\lambda_{i} t_{*}}}{\alpha \eta_{i}+e^{-\lambda_{i} T}}\right)\left(f, q_{i}\right) q_{i},
$$

where the "regularization weights" $\eta_{i}$ are given by

$$
\eta_{i}=1
$$

for $(7)$, and

$$
\eta_{i}=e^{\lambda_{i}\left(T-2 t_{*}\right)}=e^{\lambda_{i} T(1-2 \theta)}
$$

for (9).

Now, denote the norm of the regularization error associated with $(7)$ as $\delta$, and the norm of regularization error associated with (9) as $\bar{\delta}$, so that we have (recall that $\left.b_{i}=\left(f, q_{i}\right)\right)$

$$
\delta^{2}=\left\|\phi-\phi_{\alpha}\right\|^{2}=\sum_{i=1}^{\infty} b_{i}^{2} e^{2 \lambda_{i}\left(T-t_{*}\right)}\left[\frac{\alpha}{\alpha+e^{-\lambda_{i} T}}\right]^{2},
$$

using $\phi_{\alpha}$ from (7), and

$$
\bar{\delta}^{2}=\left\|\phi-\phi_{\alpha}\right\|^{2}=\sum_{i=1}^{\infty} b_{i}^{2} e^{2 \lambda_{i}\left(T-t_{*}\right)}\left[\frac{\alpha \eta_{i}}{\alpha \eta_{i}+e^{-\lambda_{i} T}}\right]^{2},
$$

using $\phi_{\alpha}$ from (9). Then we have the following result.

Theorem 6. If $\theta<\frac{1}{2}$, then $\delta<\bar{\delta}$; if $\theta>\frac{1}{2}$, then $\delta>\bar{\delta}$. Thus, in the absence of noise in the data, the regularization (7) is more accurate for long-term problems, i.e., for $\theta<\frac{1}{2}$.

Proof. This is done by a simple calculation working with the squares of the errors. We have that the difference in regularization errors is given by

$$
D=\delta^{2}-\bar{\delta}^{2}=\sum_{i=1}^{\infty} b_{i}^{2} e^{2 \lambda_{i}\left(T-t_{*}\right)}\left[\left(\frac{\alpha}{\alpha+e^{-\lambda_{i} T}}\right)^{2}-\left(\frac{\alpha \eta_{i}}{\alpha \eta_{i}+e^{-\lambda_{i} T}}\right)^{2}\right] .
$$


But the bracketed term simplifies as follows:

$$
\left(\frac{\alpha}{\alpha+e^{-\lambda_{i} T}}\right)^{2}-\left(\frac{\alpha \eta_{i}}{\alpha \eta_{i}+e^{-\lambda_{i} T}}\right)^{2}=\frac{\alpha^{2} e^{-2 \lambda_{i} T}\left(1-\eta_{i}^{2}\right)+2 \alpha^{3} \eta_{i} e^{-\lambda_{i} T}\left(1-\eta_{i}\right)}{\left(\alpha+e^{-\lambda_{i} T}\right)^{2}\left(\alpha \eta_{i}+e^{-\lambda_{i} T}\right)^{2}}
$$

hence, $D<0$ (or, $\delta^{2}<\bar{\delta}^{2}$ ) iff $\eta_{i}>1$ iff $\theta<\frac{1}{2}$, and we are done.

Remark. Note that we now have the interesting result that for any value of $\theta$, the more accurate regularization will be more sensitive to noise in the data. This is in fact borne out by the examples that we present later in the paper.

Finally, we note that (9) implies that we can write the regularization from [1] as the solution of the operator equation

$$
\alpha \mathcal{K}_{3} \phi_{\alpha}+\mathcal{K}_{1} \phi_{\alpha}=\mathcal{K}_{2} f
$$

where

$$
\mathcal{K}_{3} \psi=\sum_{i=1}^{\infty}\left(\psi, q_{i}\right) e^{\lambda_{i}\left(T-2 t_{*}\right)} q_{i}
$$

where we are implicitly assuming the sum converges. Thus both regularizations can be written as solutions of the operator equation

$$
\alpha \mathcal{L} \phi_{\alpha}+\mathcal{K}_{1} \phi_{\alpha}=\mathcal{K}_{2} f
$$

where $\mathcal{L}=I$, the identity for our regularization, and $\mathcal{L}=\mathcal{K}_{3}$ for that used in [1]. However, a better way to write the regularization from [1] is in the form

$$
\alpha \phi_{\alpha}+\mathcal{K}_{4}^{*} \mathcal{K}_{4} \phi_{\alpha}=\mathcal{K}_{4}^{*} f
$$

where

$$
\mathcal{K}_{4} \psi=\sum_{i=1}^{\infty}\left(\psi, q_{i}\right) e^{-\lambda_{i}\left(T-t_{*}\right)} q_{i}
$$

This will be needed in $\S 6$, where we do a comparison of error estimates.

\section{Numerical approximation Via EigenValues}

We now turn our attention to the problem of numerically approximating the solution to (QBVP), using an (approximate) eigenfunction expansion. Since $S(t)=$ $e^{-t A}$ we can work directly with the eigenpairs of the operator $A$, instead of having to compute the kernel function $K$ itself. Iterative techniques, which are slightly more efficient, are discussed in the next section. Much of what we do here follows $\S 3.1$ of $[1]$.

We can write out the regularized solution in terms of the eigenbasis as

$$
\phi_{\alpha}=\sum_{i=1}^{\infty}\left(\frac{e^{-\lambda_{i} t_{*}}}{\alpha+e^{-\lambda_{i} T}}\right)\left(f, q_{i}\right) q_{i} .
$$

Suppose now that we have a finite-dimensional approximate eigenbasis, i.e., a set of pairs $\left(\lambda_{i}^{h}, q_{i}^{h}\right)_{i=1}^{i=N}$, where

$$
\lambda_{i}^{h} \approx \lambda_{i}, \quad q_{i}^{h} \approx q_{i}, \quad 1 \leq i \leq N .
$$

We can then define the approximate regularized solution as

$$
\phi_{\alpha, h}=\sum_{i=1}^{N}\left(\frac{e^{-\lambda_{i}^{h} t_{*}}}{\alpha+e^{-\lambda_{i}^{h} T}}\right)\left(f, q_{i}^{h}\right) q_{i}^{h} .
$$


If we define the approximate eigenpairs via classical finite element methods, then we have

$$
a\left(q_{i}^{h}, w_{h}\right)=\lambda_{i}^{h}\left(q_{i}^{h}, w_{h}\right) \quad \forall w_{h} \in S_{0}^{h}(\Omega) \subset H_{0}^{1}(\Omega),
$$

where $S_{0}^{h}(\Omega)$ is a finite element approximating subspace [2] and $a(\cdot, \cdot)$ is the bilinear form associated with the operator $-A$. We can thus approximate the solution of (QBVP) by solving the matrix eigenvalue problem derived from (12) and then computing the approximate solution via (11).

As a matter of fact, any set of approximate eigenpairs can be used in (11); however, as we shall see in $\S 6$, the accuracy of the approximation is closely tied to the approximation properties of the subspace $S_{0}^{h}(\Omega)$.

From (11) and (1) we can easily show that

$$
\alpha \phi_{\alpha, h}+\sum_{i=1}^{N} e^{-\lambda_{i}^{h} T}\left(\phi_{\alpha, h}, q_{i}^{h}\right) q_{i}^{h}=\sum_{i=1}^{N} e^{-\lambda_{i}^{h} t_{*}}\left(f, q_{i}^{h}\right) q_{i}^{h}
$$

from which we get the definitions of the approximate operators

$$
\begin{aligned}
& \mathcal{K}_{1, h} v=\sum_{i=1}^{N} e^{-\lambda_{i}^{h} T}\left(v, q_{i}^{h}\right) q_{i}^{h}, \\
& \mathcal{K}_{2, h} v=\sum_{i=1}^{N} e^{-\lambda_{i}^{h} t_{*}}\left(v, q_{i}^{h}\right) q_{i}^{h} .
\end{aligned}
$$

We note in passing that if $v_{h}=\mathcal{K}_{j, h} v, j=1,2$, then $v_{h}$ is a semi-discrete finiteelement solution to a forward self-adjoint parabolic problem; hence the results of [3] apply. They will be needed in $\S 6$, where we derive error estimates. We also define the approximate operator needed for the regularization from [1]

$$
\mathcal{K}_{4, h} v=\sum_{i=1}^{N} e^{-\lambda_{i}^{h}\left(T-t_{*}\right)}\left(v, q_{i}^{h}\right) q_{i}^{h} .
$$

The one drawback of this method is that the discrete eigenvalue problem can be quite large, especially when the problem is posed in $\mathbf{R}^{2}$ or $\mathbf{R}^{3}$. For this reason we are led to consider iterative methods, as in the next section. However, the presence of the decaying exponentials in the approximate expansion coefficients implies that we can sometimes truncate the expansion. If we let $\epsilon$ be a truncation tolerance, then we would ignore all terms such that

$$
\left|\frac{\xi^{\theta}}{\alpha+\xi}\left(f, q_{h}^{i}\right)\right| \leq \epsilon, \theta=t_{*} / T
$$

where $\xi=e^{-\lambda_{i}^{h} T}$. The presence of the fraction $\theta$ makes explicit computation of a sharp bound for $\lambda_{i}^{h}$ difficult. However, if $\frac{1}{2} \leq \theta \leq 1$, then

$$
\left|\frac{\xi^{\theta}}{\alpha+\xi}\left(f, q_{h}^{i}\right)\right| \leq\left|\frac{\xi^{1 / 2}}{\alpha+\xi}\left(f, q_{h}^{i}\right)\right|
$$


from which we can get the (sufficient) condition that

$$
\begin{aligned}
\lambda_{i}^{h} & \geq \frac{2}{T} \log \left(\frac{2 \epsilon}{\left|\left(f, q_{i}^{h}\right)\right|-\sqrt{\left|\left(f, q_{i}^{h}\right)\right|^{2}-4 \alpha \epsilon^{2}}}\right) \\
& =\frac{2}{T} \log \left(\frac{1}{2 \alpha \epsilon}\left[\left|\left(f, q_{i}^{h}\right)\right|+\sqrt{\left|\left(f, q_{i}^{h}\right)\right|^{2}-4 \alpha \epsilon^{2}}\right]\right) .
\end{aligned}
$$

Alternatively, if $0 \leq \theta \leq \frac{1}{2}$, then

$$
\left|\frac{\xi^{\theta}}{\alpha+\xi}\left(f, q_{h}^{i}\right)\right| \leq\left|\alpha^{-1} \xi^{\theta}\left(f, q_{h}^{i}\right)\right|
$$

from which we get the (again, sufficient) condition that

$$
\lambda_{i}^{h} \geq \frac{1}{t_{*}} \log \left(\frac{\left|\left(f, q_{i}^{h}\right)\right|}{\alpha \epsilon}\right) .
$$

The practical implementation of this truncation scheme is discussed in $\S 7$.

\section{Numerical approximation Via CONJUGate GRAdient iteration}

Perhaps the best iteration technique to use for solving (1) is the standard conjugate gradient method [5], [7], [12], which converges very quickly for this problem, even in the absence of pre-conditioning. This is based on the work of Winther [15], who studied CG as applied to operators of the form $I+A$, where $A$ is compact. It is a very short step from this case to ours, in which the coefficient operator is $\alpha I+\mathcal{K}_{1}$, with $\mathcal{K}_{1}$ compact. Since many of the details are in [1], we will only summarize here.

Winther's main result is that the error in a CG iteration for problems governed by $I+A, A$ compact, is governed by

$$
\left\|\psi-\psi_{n}\right\| \leq\left(c_{n}\right)^{n}\left\|\psi-\psi_{0}\right\|,
$$

where $c_{n} \longrightarrow 0$ as $n \longrightarrow \infty$, thus producing superlinear convergence. The precise formula for $c_{n}$ is [1]

$$
c_{n}=\left[\left(1+\alpha^{-1} e^{-\lambda_{1} T}\right) \prod_{k=1}^{n}\left(\frac{e^{-\lambda_{k} T}}{1+\alpha e^{\lambda_{k} T}}\right)\right]^{1 / n},
$$

which clearly goes to 0 as $n$ goes to $\infty$.

To apply CG to our problem requires only that we construct some approximation to the compact operator $\mathcal{K}_{1}$. As in [1], we note that this does not require a priori knowledge of the kernel of the PDE, since we really do not need to know the operator so much as the operation, i.e., we need to know how to approximate $\mathcal{K}_{1} w$ for a given $w$. But this is nothing more than the solution to the forward parabolic problem corresponding to (FVP), so that any finite difference or finite element or spectral method will suffice. The error estimate (13) becomes

$$
\left\|\phi_{\alpha, h}-\phi_{\alpha, h}^{n}\right\| \leq\left(c_{n}\right)^{n}\left\|\phi_{\alpha, h}-\phi_{\alpha, h}^{0}\right\|,
$$

where we have now written the iteration index as a superscript. The convergence rate is now given by

$$
c_{n}=\left[\left(1+\alpha^{-1} e^{-\lambda_{1}^{h} T}\right) \prod_{k=1}^{n}\left(\frac{e^{-\lambda_{k}^{h} T}}{1+\alpha e_{k}^{\lambda_{k}^{h} T}}\right)\right]^{1 / n},
$$


where $\lambda_{k}^{h}$ is the $k^{\text {th }}$ eigenvalue of the approximate form of $\mathcal{K}_{1}$. Again, this decays rather rapidly to 0 .

\section{ERror estimates}

Having constructed the numerical approximations, we now turn our attention to estimating the error in each method.

To facilitate discussion of the error estimates, we first give and discuss a set of assumptions to be made (and, in some cases, eventually proved).

(A1) Subspace Approximation: Here we assume that the approximate solution is an element of a subspace $S^{h, d}(\Omega) \subset L^{2}(\Omega)$ with the following property: for every function $\psi \in H^{s}(\Omega)$, there is a function $\psi_{I} \in S^{h, d}(\Omega)$ such that

$$
\left\|\psi-\psi_{I}\right\| \leq C h^{\mu}\|\psi\|_{r}, \quad \mu=\min (d+1, r), \quad 0 \leq r \leq s .
$$

This is a standard result for finite-element approximations [2]. In that context, the parameter $d$ is the degree of polynomial approximation used in constructing the subspace. In the classic case of piecewise linear approximations, we have $d=1$ so (14) reduces to

$$
\left\|\psi-\psi_{I}\right\| \leq C h^{2}\|\psi\|_{2} .
$$

We also note that one choice of $\psi_{I}$ (there are many) is the $L^{2}$-orthogonal projection $P_{h} \psi$ defined by:

$$
\left(\psi-P_{h} \psi, v_{h}\right)=0, \quad \forall v_{h} \in S^{h, d}(\Omega) .
$$

(A2) Operator Approximation: Here we assume that the approximate operators $\mathcal{K}_{1, h}$ and $\mathcal{K}_{2, h}$ are accurate in the sense that there exist positive constants $C_{i}, i=1,2$ such that

$$
\left\|\left(\mathcal{K}_{i}-\mathcal{K}_{i, h}\right) v\right\| \leq C_{i} h^{\nu}\|v\|_{q}, \quad \nu=\min (d+1, q),
$$

for some $q>0$. We note in passing that since the approximate operators are essentially representations of standard approximations to forward parabolic problems, estimates such as this are well known in the literature. See, for example, Theorems $2.1,3.2$, and 3.3 of [3].

(A3) Smoothness of initial data: We say that the data function $f$ is pre-diffused by $\gamma$ if there exists a constant $M_{\gamma}$ such that

$$
\sum_{i=1}^{\infty} b_{i}^{2} e^{2 \gamma \lambda_{i}} \leq M_{\gamma}^{2}
$$

where we recall that the $b_{i}$ are the coefficients of $f$ in the eigenfunction basis. This assumption is tantamount to assuming that the initial data is the result of evolving the forward operator over a time interval of length $\gamma$, and was first used, in a slighter different form, by Miranker [9]. Note that the assumption that $\|u(0)\| \leq m$ implies that $f$ is pre-diffused by $T$.

Theorem 7. Let $\phi_{\alpha, h}$ be the approximate solution computed according to the eigenvalue technique described in $\S 4$, using the regularization (7). If (A1), (A2), and (A3) hold, with $\gamma \geq 2 T-t_{*}$, and if $\phi_{\alpha} \in H^{r}(\Omega)$, then there exists a constant $C>0$ such that

$$
\left\|\phi-\phi_{\alpha, h}\right\| \leq C\left(\alpha^{\theta} M_{\gamma}+h^{\mu}\left\|\phi_{\alpha}\right\|_{r}+m_{\alpha, h} h^{\nu}\left(\|f\|_{q}+\left\|\phi_{\alpha}\right\|_{q}\right)\right),
$$


where

$$
\theta=\left(\gamma+t_{*}-T\right) / T \geq 1
$$

and

$$
m_{\alpha, h}=\frac{\left\|e_{h}\right\|^{2}}{\alpha\left\|e_{h}\right\|^{2}+\left\|\mathcal{K}_{1, h}^{1 / 2} e_{h}\right\|^{2}}
$$

where

$$
e_{h}=P_{h} \phi_{\alpha}-\phi_{\alpha, h}
$$

Proof. We first note that the triangle inequality and Theorem 4 imply that

$$
\begin{aligned}
\left\|\phi-\phi_{\alpha, h}\right\| & \leq\left\|\phi-\phi_{\alpha}\right\|+\left\|\phi_{\alpha}-\phi_{\alpha, h}\right\| \\
& \leq \alpha^{\theta} M_{\gamma}+\left\|\phi_{\alpha}-\phi_{\alpha, h}\right\|
\end{aligned}
$$

for $\gamma$ as specified, so it only remains to bound the approximation error $\left\|\phi_{\alpha}-\phi_{\alpha, h}\right\|$. We have

$$
\begin{aligned}
\left(\mathcal{K}_{1}+\alpha I\right) \phi_{\alpha} & =\mathcal{K}_{2} f \\
\left(\mathcal{K}_{1, h}+\alpha I\right) \phi_{\alpha, h} & =\mathcal{K}_{2, h} f
\end{aligned}
$$

so that

$$
\begin{aligned}
\left(\mathcal{K}_{1, h}+\alpha I\right)\left(v_{h}-\phi_{\alpha, h}\right) & =\left(\mathcal{K}_{2}-\mathcal{K}_{2, h}\right) f \\
& +\alpha\left(v_{h}-\phi_{\alpha}\right) \\
& +\left(\mathcal{K}_{1, h} v_{h}-\mathcal{K}_{1} \phi_{\alpha}\right) .
\end{aligned}
$$

Take $v_{h}=P_{h} \phi_{\alpha}$, denote $e_{h}=P_{h} \phi_{\alpha}-\phi_{\alpha, h}$, then multiply (15) by $e_{h}$ and take inner products to get

$$
\begin{aligned}
\left(\left(\mathcal{K}_{1, h}+\alpha I\right) e_{h}, e_{h}\right) & =\left(\left(\mathcal{K}_{2}-\mathcal{K}_{2, h}\right) f, e_{h}\right) \\
& +\alpha\left(P_{h} \phi_{\alpha}-\phi_{\alpha}, e_{h}\right) \\
& +\left(\mathcal{K}_{1, h}\left(P_{h} \phi_{\alpha}-\phi_{\alpha}\right), e_{h}\right) \\
& +\left(\left(\mathcal{K}_{1} v_{h}-\mathcal{K}_{1, h} \phi_{\alpha}\right), e_{h}\right) .
\end{aligned}
$$

Taking each term on the right in turn, we get

$$
\begin{aligned}
\left|\left(\left(\mathcal{K}_{2}-\mathcal{K}_{2, h}\right) f, e_{h}\right)\right| & \leq\left\|\mathcal{K}_{2}-\mathcal{K}_{2, h}\right\|\|f\|\left\|e_{h}\right\| \\
& \leq C_{2} h^{\nu}\|f\|_{q}\left\|e_{h}\right\|
\end{aligned}
$$

by (A2);

$$
\alpha\left(P_{h} \phi_{\alpha}-\phi_{\alpha}, e_{h}\right)=0
$$

by definition of $P_{h} \phi_{\alpha}$, since $e_{h} \in S^{h, d}(\Omega)$;

$$
\left(\mathcal{K}_{1, h}\left(P_{h} \phi_{\alpha}-\phi_{\alpha}\right), e_{h}\right)=0
$$

since $\mathcal{K}_{1, h}$ is self-adjoint; and, finally,

$$
\begin{aligned}
\left|\left(\left(\mathcal{K}_{1} v_{h}-\mathcal{K}_{1, h} \phi_{\alpha}\right), e_{h}\right)\right| & \leq\left\|\mathcal{K}_{1, h}-\mathcal{K}_{1}\right\|\left\|\phi_{\alpha}\right\|\left\|e_{h}\right\| \\
& \leq C_{1} h^{\nu}\left\|\phi_{\alpha}\right\|_{q}\left\|e_{h}\right\| .
\end{aligned}
$$

At this point, then, we have

$$
\left(\left(\mathcal{K}_{1, h}+\alpha I\right) e_{h}, e_{h}\right) \leq C_{3} h^{\nu}\left(\|f\|_{q}+\left\|\phi_{\alpha}\right\|_{q}\right)\left\|e_{h}\right\| .
$$


However, the left side can be bounded below, very weakly, to get:

$$
\left(\left(\mathcal{K}_{1, h}+\alpha I\right) e_{h}, e_{h}\right)=\left(\mathcal{K}_{1, h} e_{h}, e_{h}\right)+\alpha\left(e_{h}, e_{h}\right) \geq\left(\frac{\left\|\mathcal{K}_{1, h}^{1 / 2} e_{h}\right\|^{2}}{\left\|e_{h}\right\|^{2}}+\alpha\right)\left\|e_{h}\right\|^{2} .
$$

So we have

$$
\left\|e_{h}\right\| \leq C m_{\alpha, h} h^{\nu}\left(\left\|\phi_{\alpha}\right\|_{q}+\|f\|_{q}\right) .
$$

Thus

$$
\begin{aligned}
\left\|\phi_{\alpha}-\phi_{\alpha, h}\right\| & \leq\left\|\phi_{\alpha}-P_{h} \phi_{\alpha}\right\|+\left\|P_{h} \phi_{\alpha}-\phi_{\alpha, h}\right\| \\
& \leq C m_{\alpha, h} h^{\nu}\left(\left\|\phi_{\alpha}\right\|_{q}+\|f\|_{q}\right)+C h^{\mu}\left\|\phi_{\alpha}\right\|_{r},
\end{aligned}
$$

which, combined with the regularization estimate (2), completes the proof.

Remark. If the initial data is not pre-diffused then Theorem 5 can be used to bound the regularization error, making the final estimate

$$
\left\|\phi-\phi_{\alpha, h}\right\| \leq C\left(m \alpha^{\theta}+h^{\mu}\left\|\phi_{\alpha}\right\|_{r}+m_{\alpha, h} h^{\nu}\left(\|f\|_{q}+\left\|\phi_{\alpha}\right\|_{q}\right)\right)
$$

for $\theta=t_{*} / T$.

The same basic argument can be applied to obtain an error estimate for approximating the regularization from [1]. The result is

$$
\left\|\phi-\phi_{\alpha, h}\right\| \leq C\left(\alpha^{\tau}+h^{\mu}\left\|\phi_{\alpha}\right\|_{r}+\bar{m}_{\alpha, h} h^{\nu}\left(\|f\|_{q}+\left\|\phi_{\alpha}\right\|_{q}\right)\right)
$$

for

$$
\tau=\theta /(2-\theta), \quad \theta=t_{*} / T
$$

and

$$
\bar{m}_{\alpha, h}=\frac{\left\|e_{h}\right\|^{2}}{\alpha\left\|e_{h}\right\|^{2}+\left\|\mathcal{K}_{4, h} e_{h}\right\|^{2}} .
$$

Here we have used the regularization error estimate (10) and we have assumed an approximation result for $\mathcal{K}_{4, h}$ analogous to (A2). The estimate (17) is in slightly different form than given in [1] because the proof there was based on log-convexity arguments rather than the eigenexpansion techniques used here.

The principal difference between the estimates for the two regularizations is in the $m_{\alpha, h}$ and $\bar{m}_{\alpha, h}$ coefficients. Conservative upper bounds for these are

$$
\begin{aligned}
& m_{\alpha, h} \leq \frac{1}{\alpha+e^{-\lambda_{N}^{h} T}}, \\
& \bar{m}_{\alpha, h} \leq \frac{1}{\alpha+e^{-2 \lambda_{N}^{h}\left(T-t_{*}\right)}},
\end{aligned}
$$

both of which can be bounded above by $\alpha^{-1}$.

To get a better sense of the convergence rate implied by these results, let us assume we are using linear finite elements, so that we have $d=1, r=q=\mu=\nu=2$, and also assume that $\theta=1$. The best we can do with $m_{\alpha, h}$ or $\bar{m}_{\alpha, h}$ is $m_{\alpha, h} \leq \alpha^{-1}$, which our computational experience tells us is very conservative. Then the estimate is

$$
\left\|\phi-\phi_{\alpha, h}\right\| \leq C\left(\alpha M_{\gamma}+h^{2}\left\|\phi_{\alpha}\right\|_{2}+h^{2} \alpha^{-1}\left(\|f\|_{2}+\left\|\phi_{\alpha}\right\|_{2}\right)\right) .
$$


To "balance" the error contribution from the separate terms we take $\alpha=h^{s}$ and find $s$ so that $h^{s}=h^{2-s}$. This yields $s=1$ and the final estimate is

$$
\left\|\phi-\phi_{\alpha, h}\right\| \leq C h\left(M_{\gamma}+\|f\|_{2}+\left\|\phi_{\alpha}\right\|_{2}\right) .
$$

The examples in $\S 7$ will show that this estimate is somewhat conservative in that the actual error can, on occasion, decrease more rapidly than this would indicate. However, this estimate does indicate how increased smoothness of the initial data - as governed by the size of $\gamma$-can influence the error estimate. For a general regularization error of the form $\alpha^{\theta}$, we have that the final estimate is $O\left(h^{2} \frac{\theta}{1+\theta}\right)$.

\section{EXAmple COMPUTATiOnS}

Here we simply present the results of some sample calculations which demonstrate how the method works in practice. We are particularly interested in comparing how the method discussed here compares to the method from [1]. All computations were done in Fortran 77 on a variety of Sun workstations; LAPACK routines DSTEVX and DSBEVX were used to solve the eigenproblems.

We look at four different examples. Example 1 considers the very simple backward evolution of a single Fourier mode when perturbed by random noise of different amplitudes. Example 2 looks at a similar problem consisting of several Fourier modes. Example 3 looks at the backward evolution of an initial profile generated by the forward evolution of a non-smooth profile. Finally, in Example 4 we look at the cost of computation for a two-dimensional example.

Example 1. Consider the very simple backward heat equation problem given by

$$
\begin{aligned}
u_{t} & =u_{x x}, 0<x<1, t<T, \\
u(0, t) & =0, \\
u(1, t) & =0, \\
u(x, T) & =e^{-1} \sin \pi x,
\end{aligned}
$$

which has the exact solution

$$
u(x, t)=e^{\pi^{2}(T-t)-1} \sin \pi x .
$$

Taking $T=5 / 32$ we solved this problem and measured the error at $t_{*}=1 / 32(\theta=$ $1 / 5)$ and $t_{*}=1 / 8(\theta=4 / 5)$, then compared our results with the exact solution and the method of [1] for accuracy. In all cases we used linear finite element approximations, the regularization $\alpha=h^{2}$ and the sequence of grids defined by $h=2^{-j}, 2 \leq j \leq 10, \Delta t=h / 8$. The approximation was directly constructed from the eigenexpansion, rather than using the conjugate gradient iteration, and the eigenvalue computation was truncated using the tolerance of $\epsilon=h^{3}$. To measure the sensitivity of the computations to noise, we also added random perturbations to the final data, using a maximum amplitude of $\delta=10^{-k}, k=2,4,6$. Results using the method in this paper are labeled ACEO; those based on the method of [1] are labeled AE.

Note that Tables 1-6 do indeed bear out the comparison results obtained in the paper-indeed, that is the main reason for including this example. For $\theta<\frac{1}{2}$, the $\mathrm{ACEO}$ results are superior to the AE results in the absence of any perturbations. However, when the noise is added to the data, the AE results are better. 
TABLE 1. Error results for Example $1 ; \alpha=h^{2}, \theta=1 / 5$, no perturbation.

\begin{tabular}{||c|c|c||c|c||}
\hline$h^{-1}$ & Error (ACEO) & Ratio & Error (AE) & Ratio \\
\hline 4 & $0.232449 \mathrm{D}+00$ & - & $0.3847712 \mathrm{D}+00$ & - \\
\hline 8 & $0.7273950 \mathrm{D}-01$ & 3.19 & $0.1471744 \mathrm{D}+00$ & 2.61 \\
\hline 16 & $0.1940672 \mathrm{D}-01$ & 3.75 & $0.4241154 \mathrm{D}-01$ & 3.47 \\
\hline 32 & $0.4934578 \mathrm{D}-02$ & 3.94 & $0.1102365 \mathrm{D}-01$ & 3.85 \\
\hline 64 & $0.1238937 \mathrm{D}-02$ & 3.98 & $0.2783526 \mathrm{D}-02$ & 3.96 \\
\hline 128 & $0.3100667 \mathrm{D}-03$ & 4.00 & $0.6976291 \mathrm{D}-03$ & 3.99 \\
\hline 256 & $0.7753745 \mathrm{D}-04$ & 4.00 & $0.1745168 \mathrm{D}-03$ & 4.00 \\
\hline 512 & $0.1938575 \mathrm{D}-04$ & 3.99 & $0.4363600 \mathrm{D}-04$ & 4.00 \\
\hline 1024 & $0.4850102 \mathrm{D}-05$ & 4.00 & $0.1090936 \mathrm{D}-04$ & 4.00 \\
\hline 2048 & $0.6331341 \mathrm{D}+00$ & 0.00 & $0.6331341 \mathrm{D}+00$ & 0.00 \\
\hline
\end{tabular}

TABLE 2. Error results for Example 1, using (ACEO); $\alpha=h^{2}, \theta=$ $1 / 5$, perturbation $=10^{-k}, k=2,4,6$.

\begin{tabular}{||l|c|c|c|c|c|r||}
\hline \multirow{2}{*}{$h^{-1}$} & \multicolumn{2}{|c|}{$\delta=10^{-2}$} & \multicolumn{2}{c|}{$\delta=10^{-4}$} & \multicolumn{2}{c||}{$\delta=10^{-6}$} \\
\cline { 2 - 8 } & Error & Ratio & Error & Ratio & Error & Ratio \\
\hline 4 & $0.2247218 \mathrm{D}+00$ & - & $0.2323675 \mathrm{D}+00$ & - & $0.2324442 \mathrm{D}+00$ & - \\
\hline 8 & $0.6614985 \mathrm{D}-01$ & 3.40 & $0.7264156 \mathrm{D}-01$ & 3.20 & $0.7273851 \mathrm{D}-01$ & 3.19 \\
\hline 16 & $0.4583929 \mathrm{D}-01$ & 1.44 & $0.1937951 \mathrm{D}-01$ & 3.75 & $0.1940640 \mathrm{D}-01$ & 3.75 \\
\hline 32 & $0.3360654 \mathrm{D}-01$ & 1.36 & $0.4970168 \mathrm{D}-02$ & 3.90 & $0.4934827 \mathrm{D}-02$ & 3.95 \\
\hline 64 & $0.2201172 \mathrm{D}-01$ & 1.53 & $0.1254663 \mathrm{D}-02$ & 3.96 & $0.1238902 \mathrm{D}-02$ & 3.98 \\
\hline 128 & $0.8180646 \mathrm{D}-01$ & 0.27 & $0.8674530 \mathrm{D}-03$ & 1.45 & $0.3099647 \mathrm{D}-03$ & 4.00 \\
\hline 256 & $0.7458506 \mathrm{D}+00$ & 0.11 & $0.7458786 \mathrm{D}-02$ & 0.12 & $0.1075010 \mathrm{D}-03$ & 2.87 \\
\hline 512 & $0.3551384 \mathrm{D}+01$ & 0.21 & $0.3551377 \mathrm{D}-01$ & 0.21 & $0.3555905 \mathrm{D}-03$ & 0.30 \\
\hline 1024 & $0.2328992 \mathrm{D}+01$ & 1.53 & $0.2329009 \mathrm{D}-01$ & 1.53 & $0.2331157 \mathrm{D}-03$ & 1.52 \\
\hline 2048 & $0.6331435 \mathrm{D}+00$ & 3.68 & $0.6331342 \mathrm{D}+00$ & 0.04 & $0.6331341 \mathrm{D}+00$ & 0.00 \\
\hline
\end{tabular}

TABLE 3. Error results for Example 1, using (AE); $\alpha=h^{2}, \theta=$ $1 / 5$, perturbation $=10^{-k}, k=2,4,6$.

\begin{tabular}{||l|c|c|c|c|c|r||}
\hline \multirow{2}{*}{$h^{-1}$} & \multicolumn{2}{|c|}{$\delta=10^{-2}$} & \multicolumn{2}{c|}{$\delta=10^{-4}$} & \multicolumn{2}{c||}{$\delta=10^{-6}$} \\
\cline { 2 - 8 } & Error & Ratio & Error & Ratio & Error & Ratio \\
\hline 4 & $0.3788117 \mathrm{D}+00$ & - & $0.3847116 \mathrm{D}+00$ & - & $0.3847706 \mathrm{D}+00$ & - \\
\hline 8 & $0.1382453 \mathrm{D}+00$ & 2.74 & $0.1470851 \mathrm{D}+00$ & 2.62 & $0.1471735 \mathrm{D}+00$ & 2.61 \\
\hline 16 & $0.3933689 \mathrm{D}-01$ & 3.51 & $0.4238043 \mathrm{D}-01$ & 3.47 & $0.4241123 \mathrm{D}-01$ & 3.47 \\
\hline 32 & $0.1367326 \mathrm{D}-01$ & 2.88 & $0.1104827 \mathrm{D}-01$ & 3.84 & $0.1102389 \mathrm{D}-01$ & 3.85 \\
\hline 64 & $0.4547718 \mathrm{D}-02$ & 3.01 & $0.2780097 \mathrm{D}-02$ & 3.97 & $0.2783489 \mathrm{D}-02$ & 3.96 \\
\hline 128 & $0.1609398 \mathrm{D}-01$ & 0.28 & $0.6953793 \mathrm{D}-03$ & 4.00 & $0.6974211 \mathrm{D}-03$ & 3.99 \\
\hline 256 & $0.3160792 \mathrm{D}-01$ & 0.51 & $0.3562032 \mathrm{D}-03$ & 1.95 & $0.1744447 \mathrm{D}-03$ & 4.00 \\
\hline 512 & $0.5478467 \mathrm{D}-01$ & 0.58 & $0.5489150 \mathrm{D}-03$ & 0.65 & $0.4389440 \mathrm{D}-04$ & 3.97 \\
\hline 1024 & $0.3615845 \mathrm{D}-01$ & 1.52 & $0.3616016 \mathrm{D}-03$ & 1.52 & $0.1144602 \mathrm{D}-04$ & 3.83 \\
\hline 2048 & $0.6331434 \mathrm{D}+00$ & 0.06 & $0.6331342 \mathrm{D}+00$ & 0.00 & $0.6331341 \mathrm{D}+00$ & 0.00 \\
\hline
\end{tabular}


TABLE 4. Error results for Example $1 ; \alpha=h^{2}, \theta=4 / 5$, no perturbation.

\begin{tabular}{||c|c|c||c|c||}
\hline$h^{-1}$ & Error (ACEO) & Ratio & Error (AE) & Ratio \\
\hline 4 & $0.7965111 \mathrm{D}-01$ & - & $0.4065114 \mathrm{D}-01$ & - \\
\hline 8 & $0.2496477 \mathrm{D}-01$ & 3.18 & $0.1124311 \mathrm{D}-01$ & 3.62 \\
\hline 16 & $0.6663260 \mathrm{D}-02$ & 3.75 & $0.2887592 \mathrm{D}-02$ & 3.89 \\
\hline 32 & $0.1694452 \mathrm{D}-02$ & 3.94 & $0.7268653 \mathrm{D}-03$ & 3.97 \\
\hline 64 & $0.4254414 \mathrm{D}-03$ & 3.98 & $0.1820295 \mathrm{D}-03$ & 3.99 \\
\hline 128 & $0.1064752 \mathrm{D}-03$ & 4.00 & $0.4552698 \mathrm{D}-04$ & 4.00 \\
\hline 256 & $0.2662599 \mathrm{D}-04$ & 3.98 & $0.1138297 \mathrm{D}-04$ & 4.00 \\
\hline 512 & $0.6656943 \mathrm{D}-05$ & 3.99 & $0.2845813 \mathrm{D}-05$ & 4.00 \\
\hline 1024 & $0.1664256 \mathrm{D}-05$ & 4.00 & $0.7114511 \mathrm{D}-06$ & 4.00 \\
\hline 2048 & $0.9397974 \mathrm{D}-01$ & 0.00 & $0.9397974 \mathrm{D}-01$ & 0.00 \\
\hline
\end{tabular}

TABLE 5. Error results for Example 1, using (ACEO); $\alpha=h^{2}, \theta=$ $4 / 5$, perturbation $=10^{-k}, k=2,4,6$.

\begin{tabular}{||l|c|c|c|c|c|r||}
\hline \multirow{2}{*}{$h^{-1}$} & \multicolumn{2}{|c|}{$\delta=10^{-2}$} & \multicolumn{2}{c||}{$\delta=10^{-4}$} & \multicolumn{2}{c||}{$\delta=10^{-6}$} \\
\cline { 2 - 7 } & Error & Ratio & Error & Ratio & Error & Ratio \\
\hline 4 & $0.7643459 \mathrm{D}-01$ & - & $0.7961894 \mathrm{D}-01$ & - & $0.7965079 \mathrm{D}-01$ & - \\
\hline 8 & $0.2103312 \mathrm{D}-01$ & 3.63 & $0.2492537 \mathrm{D}-01$ & 3.19 & $0.2496438 \mathrm{D}-01$ & 3.19 \\
\hline 16 & $0.5501156 \mathrm{D}-02$ & 3.82 & $0.6650565 \mathrm{D}-02$ & 3.75 & $0.6663133 \mathrm{D}-02$ & 3.75 \\
\hline 32 & $0.2780416 \mathrm{D}-02$ & 1.98 & $0.1704295 \mathrm{D}-02$ & 3.90 & $0.1694551 \mathrm{D}-02$ & 3.93 \\
\hline 64 & $0.5603345 \mathrm{D}-03$ & 4.96 & $0.4240012 \mathrm{D}-03$ & 4.02 & $0.4254267 \mathrm{D}-03$ & 3.98 \\
\hline 128 & $0.1109020 \mathrm{D}-02$ & 0.51 & $0.9850982 \mathrm{D}-04$ & 4.30 & $0.1063920 \mathrm{D}-03$ & 4.00 \\
\hline 256 & $0.1083626 \mathrm{D}-02$ & 1.02 & $0.2481639 \mathrm{D}-04$ & 3.97 & $0.2658627 \mathrm{D}-04$ & 4.00 \\
\hline 512 & $0.1715889 \mathrm{D}-02$ & 0.63 & $0.1715728 \mathrm{D}-04$ & 1.45 & $0.6625423 \mathrm{D}-05$ & 4.01 \\
\hline 1024 & $0.1032948 \mathrm{D}-02$ & 1.66 & $0.1014695 \mathrm{D}-04$ & 1.69 & $0.1647635 \mathrm{D}-05$ & 4.02 \\
\hline 2048 & $0.9398951 \mathrm{D}-01$ & 0.01 & $0.9397983 \mathrm{D}-01$ & 0.00 & $0.9397974 \mathrm{D}-01$ & 0.00 \\
\hline
\end{tabular}

TABLE 6. Error results for Example 1, using (AE); $\alpha=h^{2}, \theta=$ $4 / 5$, perturbation $=10^{-k}, k=2,4,6$.

\begin{tabular}{||l|c|c|c|c|c|r||}
\hline \multirow{2}{*}{$h^{-1}$} & \multicolumn{2}{|c|}{$\delta=10^{-2}$} & \multicolumn{2}{c||}{$\delta=10^{-4}$} & \multicolumn{2}{c||}{$\delta=10^{-6}$} \\
\cline { 2 - 7 } & Error & Ratio & Error & Ratio & Error & Ratio \\
\hline 4 & $0.3699124 \mathrm{D}-01$ & - & $0.4061440 \mathrm{D}-01$ & - & $0.4065077 \mathrm{D}-01$ & - \\
\hline 8 & $0.7857295 \mathrm{D}-02$ & 4.71 & $0.1120212 \mathrm{D}-01$ & 3.63 & $0.1124270 \mathrm{D}-01$ & 3.62 \\
\hline 16 & $0.5241586 \mathrm{D}-02$ & 1.50 & $0.2875184 \mathrm{D}-02$ & 3.90 & $0.2887464 \mathrm{D}-02$ & 3.89 \\
\hline 32 & $0.3235569 \mathrm{D}-02$ & 1.62 & $0.7372301 \mathrm{D}-03$ & 3.90 & $0.7269639 \mathrm{D}-03$ & 3.97 \\
\hline 64 & $0.7371852 \mathrm{D}-03$ & 4.39 & $0.1807105 \mathrm{D}-03$ & 4.08 & $0.1820148 \mathrm{D}-03$ & 3.99 \\
\hline 128 & $0.1216247 \mathrm{D}-02$ & 0.61 & $0.3834107 \mathrm{D}-04$ & 4.71 & $0.4544383 \mathrm{D}-04$ & 4.01 \\
\hline 256 & $0.5642036 \mathrm{D}-01$ & 0.02 & $0.5642391 \mathrm{D}-03$ & 0.07 & $0.1266888 \mathrm{D}-04$ & 3.59 \\
\hline 512 & $0.1781688 \mathrm{D}-01$ & 3.17 & $0.1781387 \mathrm{D}-03$ & 3.17 & $0.3329142 \mathrm{D}-05$ & 3.81 \\
\hline 1024 & $0.2526248 \mathrm{D}-01$ & 0.71 & $0.2526208 \mathrm{D}-03$ & 0.71 & $0.2619674 \mathrm{D}-05$ & 1.27 \\
\hline 2048 & $0.9398917 \mathrm{D}-01$ & 0.27 & $0.9397983 \mathrm{D}-01$ & 0.00 & $0.9397974 \mathrm{D}-01$ & 0.00 \\
\hline
\end{tabular}


Example 2. Here we consider the same PDE, but with a slightly more involved data function. Define

$$
f(x)=e^{-\tau \pi^{2}} \sin \pi x+e^{-4 \tau \pi^{2}} \sin 2 \pi x+e^{-2-9 \tau \pi^{2}} \sin 3 \pi x
$$

from which we get that the exact solution is

$$
\begin{aligned}
u(x, t)= & e^{-\tau \pi^{2}+(T-t) \pi^{2}} \sin \pi x \\
& +e^{-4 \tau \pi^{2}+4(T-t) \pi^{2}} \sin 2 \pi x+e^{-2-9 \tau \pi^{2}+9(T-t) \pi^{2}} \sin 3 \pi x .
\end{aligned}
$$

Taking $\tau=T-t_{*}$ produces the exact solution

$$
\phi(x)=u\left(x, t_{*}\right)=\sin \pi x+\sin 2 \pi x+e^{-2} \sin 3 \pi x .
$$

Figure 1 shows the data profile and solution profiles for $T=1 / 8, t_{*}=1 / 32(\theta=$ $1 / 4)$ and $t_{*}=3 / 32(\theta=3 / 4)$. Tables 7 and 8 show the same information as in Example 1 for the numerical approximations. Two things to note are that the comparison results of $\S 3$ are again confirmed, but the errors are substantially higher than in Example 1.

TABLE 7. Error results for Example $2 ; \alpha=h^{2}, \theta=1 / 4$, no perturbation.

\begin{tabular}{||c|c|c||c|c||}
\hline$h^{-1}$ & Error (ACEO) & Ratio & Error $(\mathrm{AE})$ & Ratio \\
\hline 4 & $0.651311 \mathrm{E}+00$ & - & $0.731592 \mathrm{E}+00$ & - \\
\hline 8 & $0.500437 \mathrm{E}+00$ & 1.3010 & $0.685975 \mathrm{E}+00$ & 1.0660 \\
\hline 16 & $0.277570 \mathrm{E}+00$ & 1.8030 & $0.616086 \mathrm{E}+00$ & 1.1130 \\
\hline 32 & $0.130830 \mathrm{E}+00$ & 2.1220 & $0.444974 \mathrm{E}+00$ & 1.3850 \\
\hline 64 & $0.935625 \mathrm{E}-01$ & 1.3980 & $0.224220 \mathrm{E}+00$ & 1.9850 \\
\hline 128 & $0.770219 \mathrm{E}-01$ & 1.2150 & $0.115449 \mathrm{E}+00$ & 1.9420 \\
\hline 256 & $0.482003 \mathrm{E}-01$ & 1.5980 & $0.969024 \mathrm{E}-01$ & 1.1910 \\
\hline 512 & $0.193557 \mathrm{E}-01$ & 2.4900 & $0.943557 \mathrm{E}-01$ & 1.0270 \\
\hline 1024 & $0.570397 \mathrm{E}-02$ & 3.3930 & $0.901759 \mathrm{E}-01$ & 1.0460 \\
\hline 2048 & $0.149270 \mathrm{E}-02$ & 3.8210 & $0.768532 \mathrm{E}-01$ & 1.1730 \\
\hline
\end{tabular}

TABLE 8. Error results for Example 2; $\alpha=h^{2}, \theta=3 / 4$, no perturbation.

\begin{tabular}{||c|c|c||c|c||}
\hline$h^{-1}$ & Error (ACEO) & Ratio & Error (AE) & Ratio \\
\hline 4 & $0.601714 \mathrm{E}+00$ & - & $0.344687 \mathrm{E}+00$ & - \\
\hline 8 & $0.471825 \mathrm{E}+00$ & 1.2750 & $0.155881 \mathrm{E}+00$ & 2.2110 \\
\hline 16 & $0.264104 \mathrm{E}+00$ & 1.7870 & $0.633696 \mathrm{E}-01$ & 2.4600 \\
\hline 32 & $0.127430 \mathrm{E}+00$ & 2.0730 & $0.228905 \mathrm{E}-01$ & 2.7680 \\
\hline 64 & $0.931529 \mathrm{E}-01$ & 1.3680 & $0.665477 \mathrm{E}-02$ & 3.4400 \\
\hline 128 & $0.769460 \mathrm{E}-01$ & 1.2110 & $0.173857 \mathrm{E}-02$ & 3.8280 \\
\hline 256 & $0.481676 \mathrm{E}-01$ & 1.5970 & $0.439658 \mathrm{E}-03$ & 3.9540 \\
\hline 512 & $0.193434 \mathrm{E}-01$ & 2.4900 & $0.110234 \mathrm{E}-03$ & 3.9880 \\
\hline 1024 & $0.570037 \mathrm{E}-02$ & 3.3930 & $0.275784 \mathrm{E}-04$ & 3.9970 \\
\hline 2048 & $0.149177 \mathrm{E}-02$ & 3.8210 & $0.689587 \mathrm{E}-05$ & 3.9990 \\
\hline
\end{tabular}



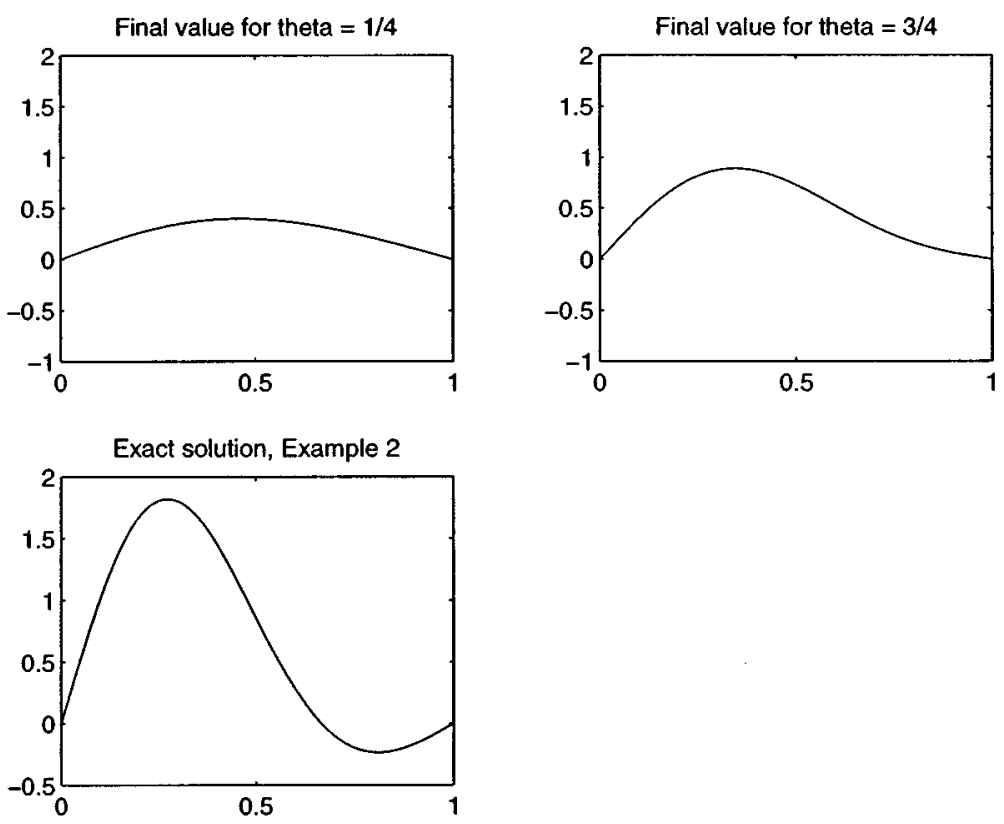

Figure 1. Profiles for Example 2

Example 3. For our third example we took the piecewise linear function

$$
v_{0}(x)= \begin{cases}0, & 0 \leq x \leq \frac{1}{2} \\ 16 x-8, & \frac{1}{2} \leq x \leq \frac{3}{4} \\ 16-16 x, & \frac{3}{4} \leq x \leq 1\end{cases}
$$

and evolved it forward in time, using the heat equation, to $t=\frac{1}{32}, \frac{1}{16}, \frac{3}{32}$, and $\frac{1}{8}$.

We then considered two problems for the backward heat equation, one defined by taking

$$
f(x)=v\left(x, \frac{1}{8}\right), \quad t_{*}=\frac{3}{32} \Longrightarrow \theta=\frac{3}{4}, \quad \phi=v\left(\cdot, \frac{3}{32}\right),
$$

and the other defined by taking

$$
f(x)=v\left(x, \frac{1}{8}\right), \quad t_{*}=\frac{1}{32} \Longrightarrow \theta=\frac{1}{4}, \quad \phi=v\left(\cdot, \frac{1}{32}\right) .
$$

Figure 2 shows the initial profile (20), the final value profile $f(x)$, and the two "exact solutions".

We note that the comparative accuracy of the two methods is the same as was observed for the first example. Also, since the exact solution is not nearly as smooth as in Example 1, we expect less accuracy in general; this is also borne out. For this reason we did not perturb the data with the random noise profile, as was done in the first example. 
TABLE 9. Error results for Example $3 ; \alpha=h^{2}, \theta=1 / 4$, no perturbation.

\begin{tabular}{||c|c|c||c|c||}
\hline$h^{-1}$ & Error (ACEO) & Ratio & Error (AE) & Ratio \\
\hline 4 & $0.3315377 \mathrm{D}+00$ & - & $0.3904057 \mathrm{D}+00$ & - \\
\hline 8 & $0.2380698 \mathrm{D}+00$ & 1.39 & $0.3286736 \mathrm{D}+00$ & 1.19 \\
\hline 16 & $0.1292539 \mathrm{D}+00$ & 1.84 & $0.2903586 \mathrm{D}+00$ & 1.13 \\
\hline 32 & $0.5715822 \mathrm{D}-01$ & 2.26 & $0.2087561 \mathrm{D}+00$ & 1.39 \\
\hline 64 & $0.3799415 \mathrm{D}-01$ & 1.50 & $0.1031234 \mathrm{D}+00$ & 2.02 \\
\hline 128 & $0.3087935 \mathrm{D}-01$ & 1.23 & $0.4897861 \mathrm{D}-01$ & 2.11 \\
\hline 256 & $0.1929986 \mathrm{D}-01$ & 1.60 & $0.3904010 \mathrm{D}-01$ & 1.25 \\
\hline 512 & $0.7747773 \mathrm{D}-02$ & 2.49 & $0.3779288 \mathrm{D}-01$ & 1.03 \\
\hline 1024 & $0.2282331 \mathrm{D}-02$ & 3.39 & $0.3610386 \mathrm{D}-01$ & 1.05 \\
\hline 2048 & $0.5336306 \mathrm{D}+00$ & 0.00 & $0.5336307 \mathrm{D}+00$ & 0.07 \\
\hline
\end{tabular}

TABLE 10. Error results for Example $3 ; \alpha=h^{2}, \theta=3 / 4$, no perturbation.

\begin{tabular}{||c|c|c||c|c||}
\hline$h^{-1}$ & Error (ACEO) & Ratio & Error (AE) & Ratio \\
\hline 4 & $0.7187147 \mathrm{D}-01$ & 0.00 & $0.4512433 \mathrm{D}-01$ & 0.00 \\
\hline 8 & $0.2741758 \mathrm{D}-01$ & 2.62 & $0.1312834 \mathrm{D}-01$ & 3.44 \\
\hline 16 & $0.1120489 \mathrm{D}-01$ & 2.45 & $0.3478319 \mathrm{D}-02$ & 3.77 \\
\hline 32 & $0.3694868 \mathrm{D}-02$ & 3.03 & $0.8850708 \mathrm{D}-03$ & 3.93 \\
\hline 64 & $0.1021037 \mathrm{D}-02$ & 3.62 & $0.2223288 \mathrm{D}-03$ & 3.98 \\
\hline 128 & $0.2852814 \mathrm{D}-03$ & 3.58 & $0.5564034 \mathrm{D}-04$ & 4.00 \\
\hline 256 & $0.9923097 \mathrm{D}-04$ & 2.87 & $0.1390128 \mathrm{D}-04$ & 4.00 \\
\hline 512 & $0.3418836 \mathrm{D}-04$ & 2.90 & $0.3463707 \mathrm{D}-05$ & 4.01 \\
\hline 1024 & $0.9739959 \mathrm{D}-05$ & 3.51 & $0.9516162 \mathrm{D}-06$ & 3.64 \\
\hline 2048 & $0.1019063 \mathrm{D}+00$ & 0.00 & $0.1019063 \mathrm{D}+00$ & 0.00 \\
\hline
\end{tabular}

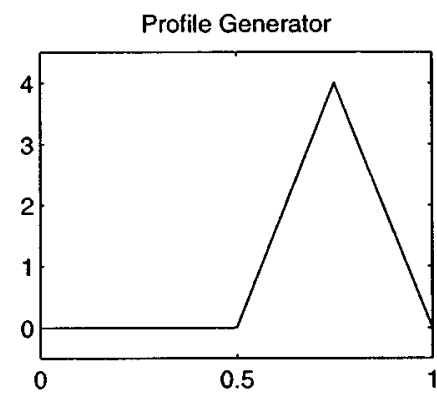

Exact solution, theta $=1 / 4$

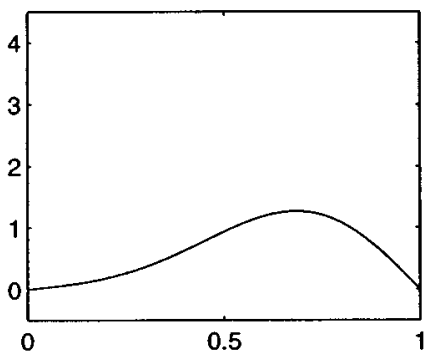

Final Value Profile

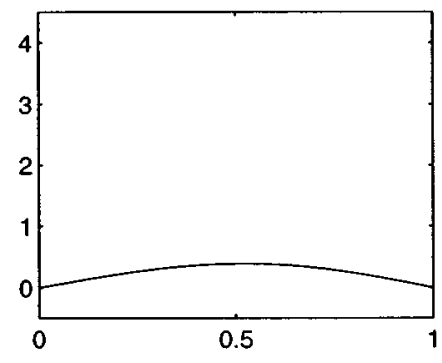

Exact solution, theta $=3 / 4$

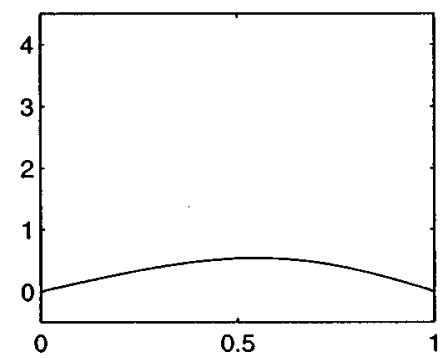

Figure 2. Profiles for Example 3 
Example 4. Our final example is a smooth two-dimensional problem. We consider the solution of the backward heat equation

$$
\begin{aligned}
u_{t} & =u_{x x}+u_{y y}, \quad(x, y) \in \Omega=(0,1) \times(0,1), t<T \\
u(x, y, t) & =0,(x, y) \in \partial \Omega \\
u(x, y, T) & =e^{-1} \sin \pi x \sin \pi y
\end{aligned}
$$

which has exact solution

$$
u(x, y, t)=e^{-1} e^{-2 \pi^{2}(t-T)} \sin \pi x \sin \pi y .
$$

We take $T=5 / 32$ and $t_{*}=1 / 8$. Our primary concern here is with the cost in time of executing the algorithm described here. Our data is summarized in Table 11. Note that the CG iteration is much more cost effective than the eigenvalue approximation, even though very few eigenvalues are being used. However, this is a little deceptive. The eigensolver used (DSBEVX from LAPACK) is a generalpurpose eigensolver for banded matrices and requires large amounts of storage even if only some of the eigenvalues/vectors are being computed. An eigensolver that was more storage efficient would almost certainly have taken substantially less CPU time.

For the eigenvalue-based approximations, we truncated the expansion when the terms became less than $h^{3}$; for the CG-based approximations, we terminated the iteration when successive iterates differed by less than $h^{3}$.

TABLE 11. Timing results in CPU seconds for Example $4 ; \alpha=$ $h^{2}, \theta=4 / 5$, no perturbation. Here $M=$ the number of eigenvalues used in the approximation, and $N=$ the number of conjugate gradient iterations needed for convergence.

\begin{tabular}{||c|c|c|c|c||c|c|c|c||}
\hline$h^{-1}$ & \multicolumn{3}{|c||}{ Eigenvalue Method } & \multicolumn{4}{c||}{ CG Method } \\
\hline & \multicolumn{2}{|c|}{ ACEO } & \multicolumn{2}{c|}{ AE } & \multicolumn{2}{c||}{ ACEO } & \multicolumn{2}{c||}{ AE } \\
\hline & Time & $M$ & Time & $M$ & Time & $N$ & Time & $N$ \\
\hline 4 & 0.050 & 8 & 0.050 & 3 & 0.010 & 2 & 0.020 & 2 \\
\hline 8 & 0.220 & 8 & 0.190 & 3 & 0.070 & 2 & 0.080 & 2 \\
\hline 16 & 11.940 & 11 & 11.600 & 4 & 0.140 & 2 & 0.310 & 2 \\
\hline 32 & 818.180 & 13 & 804.950 & 6 & 0.760 & 2 & 1.960 & 2 \\
\hline 64 & - & - & - & - & 5.360 & 2 & 13.540 & 2 \\
\hline 128 & - & - & - & - & 39.390 & 2 & 104.690 & 2 \\
\hline
\end{tabular}

\section{REFERENCES}

[1] Ames, K.A., and Epperson, J.F., A Kernel-based Method for the Approximate Solution of Backward Parabolic Problems, SIAM J. Num. Anal., Vol 34, no. 4, 1997, pp. 1357-1390. CMP 97:16

[2] Brenner, S.C., and Scott, R.S., The Mathematical Theory of Finite Element Methods, Springer-Verlag, New York, 1994. MR 95f:65001

[3] Bramble, J., Schatz, A., Thomee, V., and Wahlbin, L., Some convergence estimates for semidiscrete Galerkin approximations for parabolic equations, SIAM J. Num. Anal., 14 (1977), pp. 218-241. MR 56:7231

[4] Clark, G. and Oppenheimer, C., Quasireversibility Methods for Non-Well-Posed Problems, Elect. J. Diff. Eqns., 1994. MR 96a:34123

[5] Golub, G., and van Loan, C., Matrix Computations, Johns Hopkins University Press, Baltimore, 1983. MR 85h:65063 
[6] Groetsch, C., The theory of Tikhonov Regularization for Fredholm Equations of the First Kind, Pitman, Boston, 1984. MR 85k:45020

[7] Kelley, C.T., Iterative Methods for Linear and Nonlinear Equations, SIAM, Philadelphia, 1995. MR 96d:65002

[8] Lattes, R. and Lions, J.L., Methode de Quasi-Reversibility et Applications, Dunod, Paris, 1967 (English translation R. Bellman, Elsevier, New York, 1969) MR 38:874

[9] Miranker, W. L., A well-posed problem for the backward heat equation, Proc. Amer. Math. Soc., 12 (1961), pp. 243-247. MR 22:11216

[10] Miller, K., Stabilized quasireversibility and other nearly best possible methods for nonwell-posed problems, Symposium on Non-Well-Posed Problems and Logarithmic Convexity, Lecture Notes in Mathematics, Vol. 316, Springer-Verlag, Berlin, 1973, pp. 161-176. MR 52:14710

[11] Payne, L.E., Some general remarks on improperly posed problems for partial differential equations, Symposium on Non-Well-Posed Problems and Logarithmic Convexity, Lecture Notes in Mathematics, Vol. 316, Springer-Verlag, Berlin, 1973, pp 1-30 MR 53:13892

[12] Shewchuk, J.R., An introduction to the conjugate gradient method without the agonizing pain, electronically published manuscript.

[13] Showalter, R.E., The Final Value Problem for Evolution Equations, J. Math. Anal. Appl., 47, 1974, pp 563-572 MR 50:5131

[14] Showalter, R.E., Cauchy Problem for Hyper-Parabolic Partial Differential Equations, Trends in the Theory and Practice of Non-Linear Analysis, Elsevier, 1983 MR 87f:35121

[15] Winther, R., Some Superlinear Convergence Results for the Conjugate Gradient Method, SIAM J. Num. Anal., Vol. 17, no. 1, 1980, pp. 14-17. MR 81k:65060

Department of Mathematical Sciences, University of Alabama in Huntsville, Huntsville, Alabama 35899

E-mail address: ames@math.uah.edu

Department of Mathematics and Statistics, Mississippi State University, Drawer MA MSU, MS 39762

Current address: Department of Mathematical Sciences, Virginia Commonwealth University, Richmond, VA 23284

E-mail address: gwclark@saturn.vcu.edu

Department of Mathematical Sciences, University of Alabama in Huntsville, Huntsville, Alabama 35899

E-mail address: epperson@math.uah.edu

Department of Mathematics and Statistics, Mississippi State University, Drawer Ma MSU, MS 39762

E-mail address: seth@math.msstate.edu 